\title{
Genesis
}

Manuscrits - Recherche - Invention

$51 \mid 2020$

Intertextualité - Exogenèse

\section{L'exogenèse scientifique et science-fictionnelle chez Marie Darrieussecq : une poétique de l'escamotage}

Karine Germoni et Élise Nottet-Chedeville

\section{OpenEdition}

Journals

Édition électronique

URL : https://journals.openedition.org/genesis/5712

DOI : 10.4000/genesis. 5712

ISSN : 2268-1590

Éditeur :

Presses universitaires de Paris Sorbonne (PUPS), Société internationale de génétique artistique littéraire et scientifique (SIGALES)

\section{Édition imprimée}

Date de publication : 15 décembre 2020

Pagination : 141-148

ISBN : 979-10-231-0704-3

ISSN : $1167-5101$

\section{Référence électronique}

Karine Germoni et Élise Nottet-Chedeville, « L'exogenèse scientifique et science-fictionnelle chez Marie Darrieussecq : une poétique de l'escamotage », Genesis [En ligne], 51 | 2020, mis en ligne le 20

décembre 2021, consulté le 07 février 2022. URL : http://journals.openedition.org/genesis/5712 ; DOI : https://doi.org/10.4000/genesis.5712

Ce document a été généré automatiquement le 7 février 2022.

Tous droits réservés 


\title{
L'exogenèse scientifique et science- fictionnelle chez Marie Darrieussecq : une poétique de l'escamotage
}

\author{
Karine Germoni et Élise Nottet-Chedeville
}

Dans les fictions de Marie Darrieussecq, la présence de la science et de la science-fiction s'avère, à de rares exceptions près ${ }^{1}$, discrète, y compris dans ses œuvres de «littérature imminente ${ }^{2}$ » que sont White (2003) et Notre vie dans les forêts (2017), qu'il s'agisse de mentions explicites ou de références textuelles précises. L'auteure en reconnait pourtant l'influence dans sa démarche d'écriture : «J'ai toujours, dans ma vie privée, aimé les scientifiques et ils m'ont apporté un énorme réservoir d'images. La physique quantique est très romanesque, par exemple. Ou le paradoxe de Fermi. Et j'ai lu beaucoup de science-fiction dans mon adolescence ${ }^{3}$. » De fait, l'examen des manuscrits et des documents d'archives déposés dernièrement à l'Institut mémoires de l'édition contemporaine par Marie Darrieussecq révèle que les sources tant scientifiques que science-fictionnelles jouent un rôle matriciel dans la genèse de nombre de ses romans, bien plus important que ne le laisse paraitre l'œuvre publiée. L'analyse de ses avanttextes démontre en effet que l'écrivaine puise constamment dans ses lectures, films et documents d'archives des informations, puis se livre au fil de ses campagnes d'écriture à des procédures d'effacement de ces sources selon un continuum qui s'échelonne de la suppression totale du matériau extérieur jusqu'à sa présence diffuse, camouflée par un travail de récriture stylistique. Si l'exogenèse scientifique et science-fictionnelle fascine autant chez Darrieussecq, c'est qu'elle tisse entre les œuvres publiées des liens jusqu'alors insoupçonnés, invite pour certaines à en reconsidérer le sens et révèle un rapport dialectique qui réinterroge les notions de "propriété » et de "territorialité »", littéraires ou scientifiques, l'interaction dialogique avec les œuvres-sources ne devenant productive que lorsqu'elle s'annule et s'oublie. Ainsi, après avoir présenté quelques-uns de ces « réacteurs irradiants » que sont les sources science-fictionnelles présentes dans les carnets de lecture de jeunesse de Marie Darrieussecq et leurs 
modalités d'incorporation dans son écriture, nous étudierons le traitement réservé en diachronie à l'iridium, une donnée scientifique récurrente dans la création darrieussecquienne, plus ou moins escamotée selon les œuvres qu'elle irrigue, mais néanmoins toujours vecteur de poéticité.

\section{La science-fiction comme « réacteur irradiant »}

Lue, relue, aimée et questionnée, la science-fiction est un «réacteur irradiant sans cesse $^{5}$ » l'imaginaire de l'apprenti-écrivaine comme de l'écrivaine confirmée et reconnue. Les carnets de lecture et les manuscrits de Marie Darrieussecq sont le premier témoignage concret et vivant d'une exogenèse science-fictionnelle.

3 Tenus de 1982 à 1986 sur des cahiers d'écolier petit format, ses carnets de lecture révèlent une véritable boulimie livresque, qui se traduit par des données chiffrées. La jeune fille, alors âgée de treize à dix-sept ans, date chacun des livres lus, le commente et lui attribue une note. Ces carnets de lecture permettent de reconstituer, partiellement tout au moins, la bibliothèque science-fictionnelle virtuelle de Marie Darrieussecq : Barjavel, plusieurs anthologies de science-fiction, Le Troupeau aveugle et L'Envers du temps de John Brunner, Abattoir 5 de Kurt Vonnergut Jr... En octobre 1983, l'adolescente écrit: «Le Navire des glaces. super. Pas fini »; puis, en 1984 : «Livres préférés: La nuit des temps, Barjavel, Le voyageur imprudent». Enki Bilal est abondamment commenté pendant l'année 1986; par exemple : «Bilal (LA + Mémoires d'outre-espace : génial) »; « Bilal : Le bol maudit : excellent »...

Fig. 1 : Carnet de lectures (1982-1987), première quinzaine de février 1984, cote : 849DRQ/3221

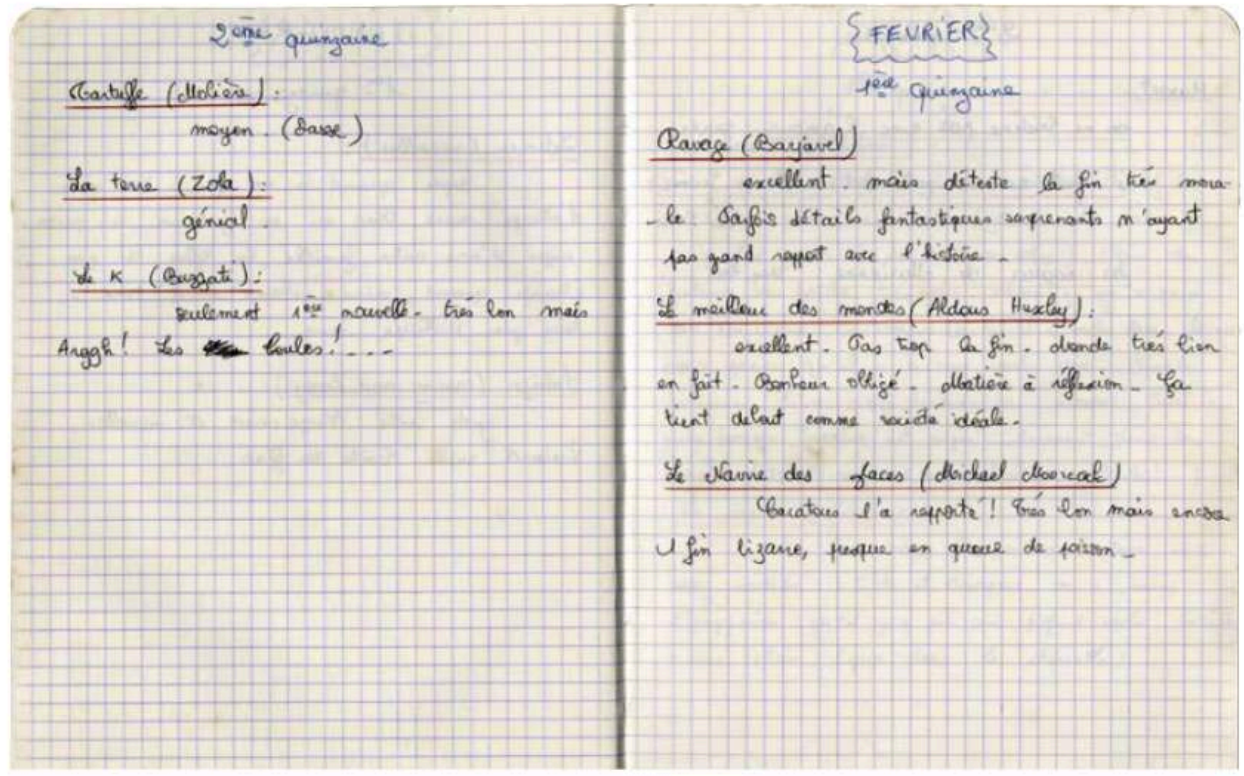

(c) IMEC/Archives Marie Darrieussecq

4 L'accès à ces notes de jeunesse confère le statut d'exogenèse science-fictionnelle darrieussecquienne à ce que la lecture de l'œuvre publiée ne permet d'envisager que comme hypothèse intertextuelle ou influence diffuse. Les clones de Notre vie dans les 
forêts apparaissent ainsi comme les avatars de ceux d'Et on tuera tous les affreux ${ }^{6}$, roman commenté avec enthousiasme en avril 1985 par la jeune fille qui adore Boris Vian. Quant à White, il est irrigué par La Nuit des temps aussi bien que par le roman Le Navire des glaces ${ }^{7}$ dont le souvenir marquant a été réactivé par d'autres sources: les cartes blanches du Pôle, le film de recherche de son mari, le physicien Jean Duprat, relatant ses expéditions au pôle Sud et plus encore les photographies prises par Franck Hurley durant l'expédition de Shackleton au pôle Sud ${ }^{8}$. Pour autant, les références à la sciencefiction sont totalement gommées dans White, ainsi que l'atteste la comparaison du manuscrit du roman avec la version publiée. Nous n'en donnerons que ce seul exemple, puisé dans le passage intitulé «III. ÉDMÉE » (p. 37 du premier cahier manuscrit ${ }^{9}$ ) qui correspond à la page 73 du roman publié dans la collection « Folio » :

[version manuscrite] On dit que c'est en Islande qu'est l'entrée vers le centre de la Terre. Par la gueule du Snaeffel, Jules Verne. Dans une bande dessinée, Spiderman ? - à Vancouver : six cases blanches. Le héros - Spiderman ? - avait été envoyé en enfer, et l'enfer c'était de n'avoir plus ni haut ni bas, ni sol ni profondeur. [version publiée] En Islande est l'entrée vers le centre de la Terre. Elle a lu ça dans une bande dessinée. Il y avait six cases blanches, où les héros se perdaient. Ils étaient en enfer et l'enfer c'était de n'avoir ni haut ni bas, ni largeur ni profondeur, ni droite ni gauche, aucun point stable.

Sont simultanément supprimées les références nominales à Jules Verne (mentionné dans les carnets de lecture), au Snæffel et à Spiderman. Si demeure une allusion claire au roman vernien à travers la mention de l'Islande et du groupe prépositionnel « vers le centre de la Terre ", la référence n'en est pas moins opacifiée. Alors que dans le manuscrit sont évoquées deux sources distinctes - le roman vernien et un épisode vancouvérois de la bande dessinée Spiderman, avec une circonspection marquée par les points d'interrogation qui ont l'air de mettre en cause autant l'exactitude de la source dans l'ordre de la fiction que la pertinence du maintien ultérieur de la référence dans l'ordre de l'écriture -, dans White, la référence à la première est gommée par la suppression de la formule "On dit que " marquant l'hétérogénéité énonciative. Le lecteur qui aura identifié l'allusion à Jules Verne pensera qu'est ici convoquée une adaptation du roman vernien en bande dessinée, le pronom «ça » prenant une valeur anaphorique. Il s'agit là d'une procédure complexe de suppression-opacification avec défiguration des sources science-fictionnelles, qui nous renvoie aux propos de PierreMarc de Biasi à propos des modalités d'incorporation des matériaux exogènes dans leur (con)texte d'accueil :

La trace de leur extériorité primitive est volatile, et à partir d'un certain point d'élaboration, la note exogénétique s'intègre si intimement à son contexte endogénétique qu'elle en devient méconnaissable. Cette conversion de l'exogénétique en endogénétique s'accomplit principalement sous l'effet de la textualisation, fonction opératoire dont l'exécution relève pour l'essentiel des brouillons ${ }^{10}$.

Force est de constater que, quel que soit le contexte, ce travail de "textualisation " opacifiant est récurrent, mutatis mutandis, dans la manière de Marie Darrieussecq et ce, depuis ses premières tentatives d'écriture. En témoigne le traitement de la référence à Enki Bilal dans «Bleu marine ", une nouvelle de jeunesse pour laquelle nous disposons de deux manuscrits, écrits à l'encre bleue puis au bic bleu, et d'un tapuscrit. La jeune fille en effet efface, entre le second manuscrit ${ }^{11}$ et le tapuscrit, une référence assez précise à l'univers du dessinateur : "La ville ressemblait de + en + au Los Angeles de Bilal. » (fig. 2) devient «La ville était ronde et chaude comme un ventre.» (p. 4) ${ }^{12}$. Par 
une opération inverse, elle introduit un peu plus bas dans le tapuscrit (p. 9) ${ }^{13}$ le nom du dessinateur, ajoutant après la phrase "La maison s'est vidée de bouquins " déjà présente dans le second manuscrit, ces précisions: «Les collections de F'murr et de Bilal, et tous les Virginia Woolf, les disques de Griffin, d'Ayler et de Coltrane ont disparu». Si cette insertion apparaît comme le corollaire de la suppression mentionnée, pour autant elle ne la compense pas. Rien n'invite à rattacher la description révisée de la ville à l'univers bilalien métonymiquement convoqué par la mention des collections de Bilal en aval. De fait, la comparaison un peu convenue «ronde et chaude comme un ventre » ne laisse en rien deviner dans l'hypotexte la référence à Bilal, à son graphisme dur et aux couleurs froides. La textualisation de la comparaison « ressemblait de + en + au Los Angeles de Bilal " gomme entièrement le matériau science-fictionnel extérieur tandis que, simultanément, l'insertion de «Bilal " constitue une forme d'hommage à un dessinateur qu'elle lit alors beaucoup et dont elle se sait la débitrice, tant les œuvres de science-fiction de Bilal que de Verne ou encore de Barjavel procèdent comme de véritables "déclencheurs de désirs ${ }^{14}$ ", à toutes les étapes de sa carrière d'écrivain. Il en va de même des sciences dites dures qui agissent sur son travail comme de véritables stimuli.

Fig. 2 : Page du carnet « Bleu marine », non folioté, 1986, cote : 849DRQ/32/5

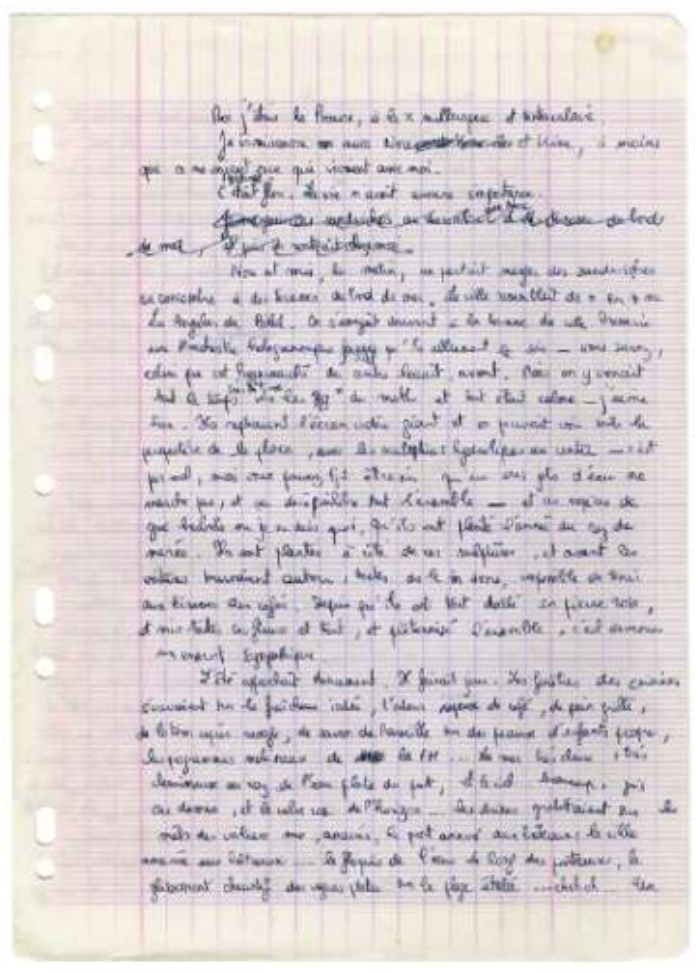

(c) IMEC/Archives Marie Darrieussecq 


\section{Un exemple d'exogenèse scientifique : l'histoire de l'iridium}

7 Si la science aiguillonne l'imaginaire darrieussecquien, c'est d'abord souvent sous la forme d'une simple discussion. C'est en conversant avec des scientifiques alors qu'elle étudie à l'École normale supérieure que la jeune femme apprend l'existence de l'iridium. Il s'agit d'un métal argenté, vraisemblablement d'origine extra-terrestre, qu'on ne trouve que dans certaines falaises - au Pays basque notamment, d'où elle vient. Marie Darrieussecq dit avoir été fascinée d'emblée par l'iridium, par le mot luimême, en raison de sa musicalité, de sa poéticité, et par son histoire, sa puissance de fiction ${ }^{15}$. De cette fascination, demeurée vivace, naît d'abord un roman écrit entre 1995 et 1996 dont le titre, Iridium $^{16}$, est éclairé par une citation documentaire en exergue, qui fonctionne pour le lecteur comme caution intellectuelle et (science-)fictionnelle et pour la romancière " comme une confirmation de [s]es intuitions ${ }^{17}{ }^{17}$ :

Une concentration exceptionnelle d'iridium [à la limite-des couches rocheuses] semble plaider, pour expliquer la fin des dinosaures et d'autres formes de vie à la fin de l'ère secondaire, en faveur de l'hypothèse souvent évoquée de l'impact d'une météorite géante. [...] En substance, la couche inférieure de rochers est constituée de calcaires marneux, et la couche supérieure de calcaires durs. La première correspond à cette période de la fin de l'ère secondaire que l'on appelle Crétacé Supérieur (ou Maastrichien, bien que la Communauté européenne ne puisse directement être mise en cause dans l'affaire qui nous occupe) et qui s'étend d'environ moins 70 à moins 65 millions d'années ${ }^{18}$.

Iridium apparaît comme le seul « véritable » récit de science-fiction de l'écrivaine : une planète imaginaire et désolée, une exploitation à outrance de l'iridium, des ressources épuisées, des gaz bleus polluants s'échappant d'une usine nucléaire, une résistance qui s'installe, un couple d'amants qui termine tragiquement au fond des glaces... Toutes les lectures science-fictionnelles de jeunesse de l'écrivaine et ses recherches sur l'iridium s'entrecroisent, se mêlent et innervent le roman en son entier.

Iridium n'est finalement pas publié. Pour autant, sa source scientifique continue de rayonner dans l'œuvre à venir. Ainsi, l'iridium, réapparaît une première fois dans Le Mal de Mer au travers de cette paraphrase poétisée de la citation de Jean-Marie Constans, délivrée de ses éléments géologiques et historiques :

L'iridium est un métal blanc venu de l'espace, sa présence sur Terre n'est due qu'à de hasardeux décrochements d'étoiles. On trouve, affleurant parfois, une unique strate contenant ces pépites à nom d'arc-en-ciel. Aucun fossile de dinosaure ne subsiste au-delà de la strate iridiée; la thèse du météorite, pour expliquer leur disparition, trouve là son principal argument ${ }^{19}$.

10 C'est ensuite dans White que l'iridium refait surface sans être directement mentionné : " Tu tranches la neige et les strates te donnent son âge, et dedans il y a des météorites, parfois. Des météorites des débuts de l'univers ${ }^{20}$. " La référence-source est ensuite glosée à trois reprises dans Le Pays en $2005^{21}$ et pour une commande "Traits traits Hermès » en 2014 dans laquelle l'écrivaine décrit un stylo offert par son éditeur argentin, dont la plume est «en iridium, un métal venu des étoiles ${ }^{22}$ " Si les résurgences de ce métal sont visibles car lisibles, et s'éclairent mutuellement, les différents états du Pays révèlent que ce matériau scientifique obsède bien plus l'écrivaine qu'on pourrait le croire. Le manuscrit du roman commencé en 2003 ne 
contenait en effet qu'une seule courte référence à l'iridium ${ }^{23}$, laquelle est ensuite doublée en 2004 par un second paragraphe sur l'iridium bien plus long et explicatif :

fig. 3 : Le Pays, 4e livret, p. 13, 18 octobre 2004, dossier " Le Pays, réécriture », cote : 849DRQ/6/1

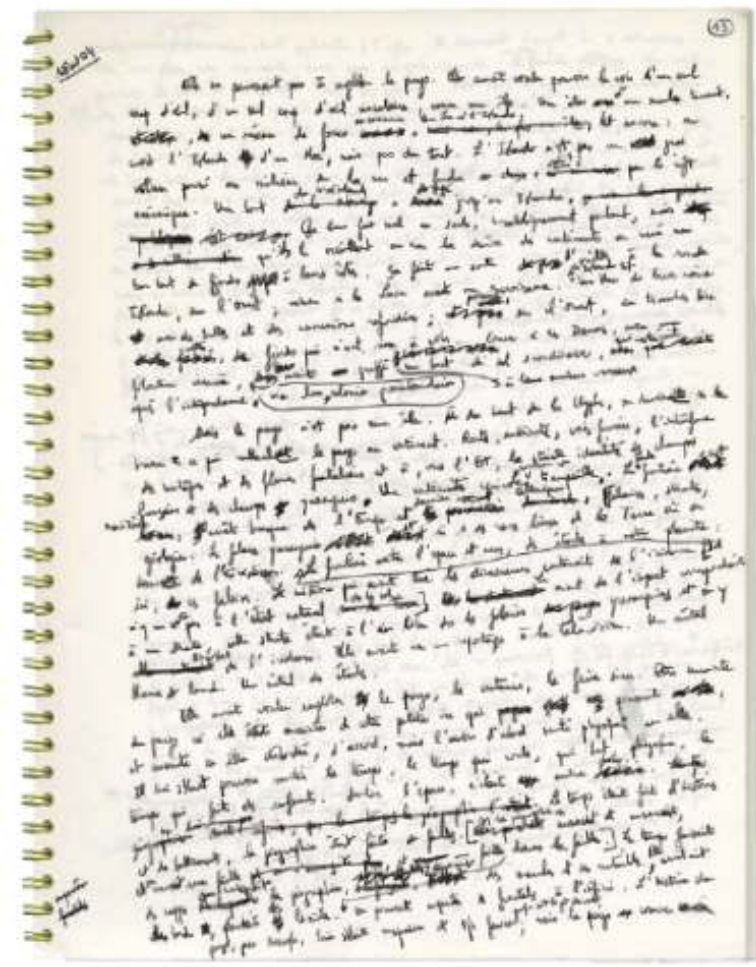

(c) IMEC/Archives Marie Darrieussecq

11 Avant de trouver sa place au début du récit ${ }^{24}$, ce paragraphe connaît plusieurs récritures : dans le premier état du tapuscrit de septembre 2004 (fig. 4 et 5), dans le deuxième état du tapuscrit en février 2005 (fig. 6) puis dans le troisième état en avril $2005^{25}$. On constate que l'auteure a supprimé les précisions géologiques et, une fois encore, amoindri les éléments qui conféraient une dimension science-fictionnelle au passage. Devenu simple mention d'un minéral présent dans la roche, le passage subit encore deux récritures sur épreuves avant de prendre sa forme définitive. 
Fig. 4 : Le Pays, tapuscrit, premier état, septembre 2004, page 38, cote : 849DRQ/6/3

Image 100E7CF800002656000035A9EE35943039ADBC14.emf

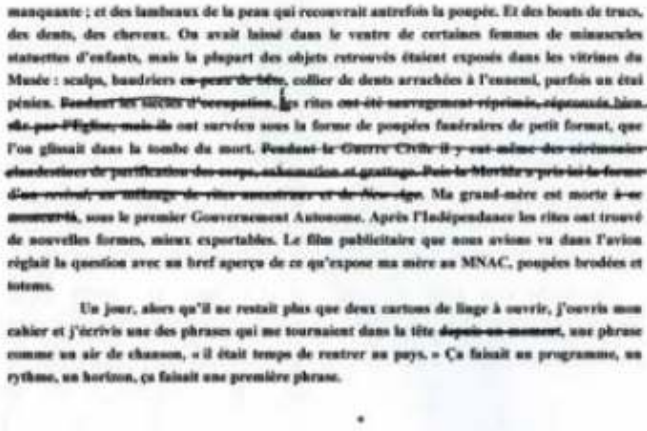

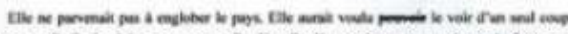

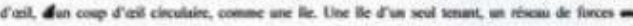

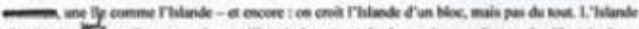

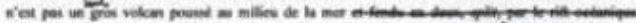

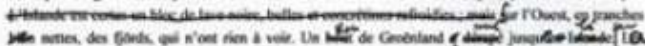

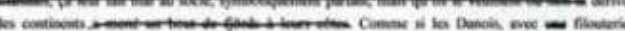

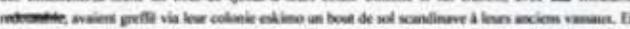

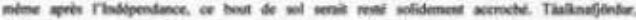

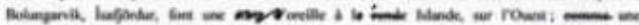
acrobsementibline.

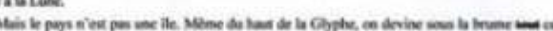

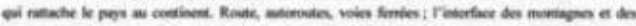

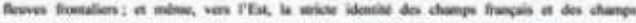

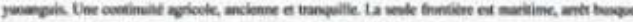

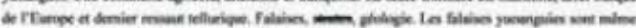

39

(C) IMEC/Archives Marie Darrieussecq

Fig. 5 : Le Pays, tapuscrit, premier état, septembre 2004, page 39, cote : 849DRQ/6/3

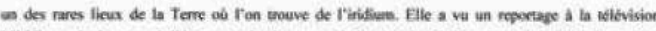

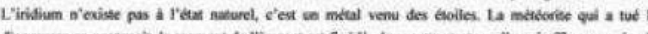

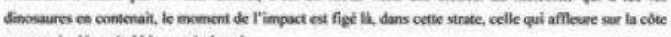
yoomguie, Uin metal blanc, vist lourd

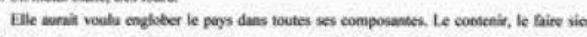

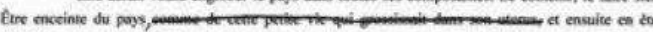

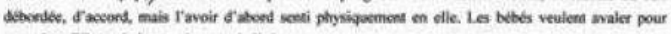

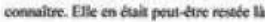

Avaat notre instaflatios, avant ce aq" on poevait appeter sotre immigratioe, at avait

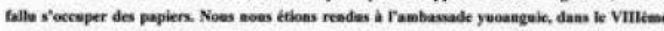

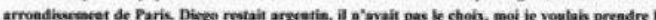

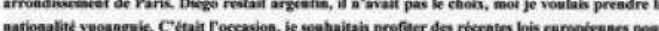

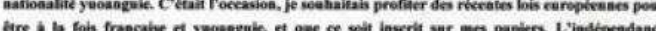
(1)

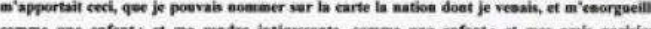

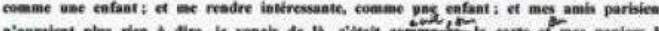

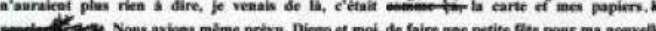

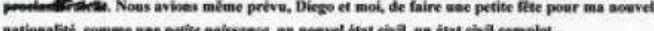

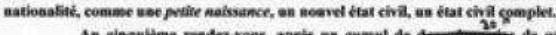

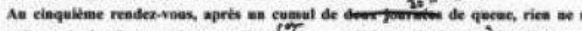

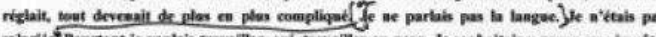

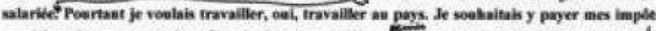

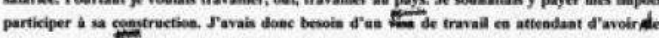

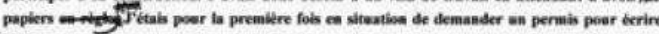

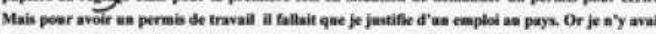

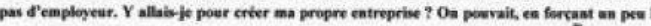

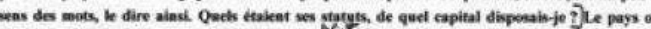

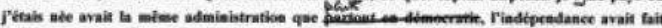

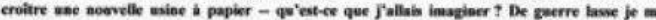

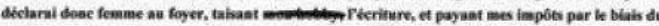

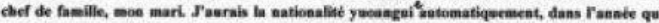

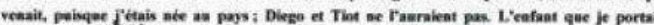

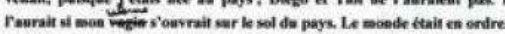


Fig. 6 : Le Pays, tapuscrit, deuxième état, février 2005, page 44, cote : 849DRQ/6/4

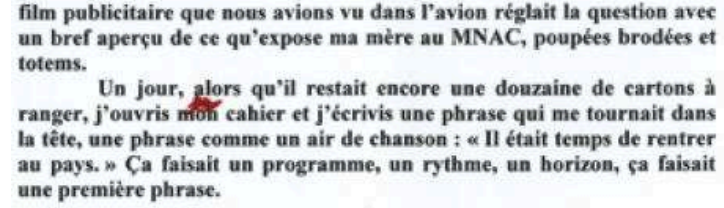

Elle ne parvenait pas a englober le pays. Elle aurait voulu le voir d'un seul coup d'œil, un coup d'eil circulaire, comme une file. Une file d'un

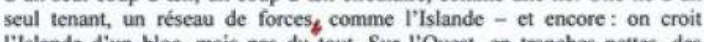
I'Islande d'un bloc, mais pas du tout. Sur l'Ouest, en tranches nettes, des fjörds allogènes débordent. Täalknafjördur, Bolungarvik, İsafjördur, font une oreille à lanndq' Islande; comme une excroissance à la Lune. On dirait un bout de Grolldand, poussé par la dérive des continents. Comme si les Danois, avec foutere, avaient greffé un bout de leur sol à leurs anciens vassaux, via leur colonie eskimo.

Mais le pays n'est pas une île. Du haut de la Glyphe, on voit les routes, autoroutes et voies ferrées, irriguter ensemble pays et continent; on voit l'interface des montagnes, la communauté dê fleuvģ; et méme, vers I'Est, la stricte identité des champs français et des champs yuoanguis: une continuité agricole, ancienne et tranquille.

La secule frontière tangible est maritime $?^{\prime}$ arrét brusque de l'Europe. Falaises, strutes, géologie. Les falaises yuoanguies sont un des lieux de la Terre oủ J'on trouve de l'iridium. Le film dans l'avion en parlait. Un métal blanc, forra, issu du métróne qui a tué les dinosaures. Le moment de l'impact est là, figé dans eélie-strateraffleurant.

Elle aurait voulu englober le pays dans toutes ses composantes. Le contenir, le faire sien, et ensuite en être débordée, d'accord, mais l'avoir d'abord senti physiquement. Les bebés veulent avaler pour connaitre. Elle en était peut-être restée li..

\section{(C) IMEC/Archives Marie Darrieussecq}

12 Si ces différents traitements de l'iridium révèlent qu'un seul terme scientifique peut agir comme moteur constant et rémanent de poéticité au sein de textes fort éloignés, ils indiquent aussi que la dynamique de l'escamotage à laquelle l'écrivaine se livre est en grande partie motivée par le souci du lecteur. De l'article de vulgarisation scientifique originel, Marie Darrieussecq ne conserve que l'infiniment paraphrasable et l'immédiatement fictionnalisable, autrement dit la part d'inexplicable. En émondant l'explicatif et en modalisant l'informatif, l'écrivaine opacifie la science au profit de la fiction et ne laisse voir qu'à l'état de traces - mentions ou allusions - sa permanente attraction pour la science-fiction.

13 Tant les carnets de lecture de jeunesse de Marie Darrieussecq que ses avant-textes nous apprennent finalement que l'imaginaire de la romancière est façonné voire hanté par des données scientifiques - telles l'iridium - et des sources science-fictionnelles (tant livresques que bédéistiques ou filmiques) qui aiguillonnent et irriguent son écriture. Ils nous apprennent plus encore que ces matériaux sont toujours travaillés sur le mode de la minoration, qu'ils soient supprimés, dissimulés, défigurés, amoindris par Darrieussecq qui les incorpore entièrement à ses récits pour qu'ils s'y fondent, conformément à sa poétique. L'écrivaine déclare en effet ne pas vouloir « digresser », la fiction n'ayant nullement la vocation d'être un documentaire ou une bibliothèque, son désir étant $\mathrm{d}^{\prime}$ " aller dans le sens du roman... et du lecteur ${ }^{26}$ » - un lecteur qu'elle dit «ne pas vouloir ennuyer » mais avec lequel elle veut néanmoins jouer, en qui l'autrice espère "une culture minimale » qui lui permette d'identifier certaines références, fussent-elles floutées ou diffuses. Ajoutons pour finir que ce masquage des sources scientifiques et science-fictionnelles révèle sur le plan de la création que Marie 
Darrieussecq n'est pas prête à assumer une écriture pleinement science-fictionnelle, alors même qu'elle en éprouve le désir depuis toujours, éprouvant très fortement le besoin d'écrire «des fictions qui ne soient pas trop éloignées du monde dans lequel [elle] vi[t $]^{27} »$.

\section{NOTES}

1. Dans Le Pays (Paris, Gallimard, coll. «Folio », 2007, p. 58) est fait mention de 1984, de Bradbury, de La Jetée et de Solaris, œuvres et auteurs commentés vingt ans auparavant dans les carnets de jeunesse.

2. Entretien de Karine Germoni et Élise Nottet-Chedeville avec Marie Darrieussecq, Paris, 26 juin 2019. Il s'agit « d'un monde parallèle légèrement décalé dans le futur ».

3. Propos recueillis en juin 2016 par l'artiste Mia Funk dans le cadre de son projet « The Creative Process ", en ligne sur creativeprocess.info.

4. Au sens que lui donnent Gilles Deleuze et Félix Guattari dans Mille Plateaux, Paris, Éditions de Minuit, 1980.

5. Expression de Marie Darrieussecq au sujet de Solaris de Stanislaw Lem et de L'Invention de Morel de Bioy Casares (courriel adressé à K. Germoni, le 4 février 2019).

6. Boris Vian (Vernon Sullivan), Et on tuera tous les affreux, (1948), Paris, Le Livre de Poche, 1997.

7. Michael Moorcock, Le Navire des glaces, (1969), Paris, Le Livre de Poche, 1978.

8. South with Endurance: Shackleton's Antarctic Expedition 1914-1917. The photographs of Franck Hurley, New York, Simon \& Schuster, 2001.

9. IMEC, fonds Marie Darrieussecq, 849DRQ5/1.

10. Pierre-Marc de Biasi, "Qu'est-ce qu'un brouillon? Le cas Flaubert, essai de typologie fonctionnelle des documents de genèse ", dans M. Contat et D. Ferrer (dir.), Pourquoi la critique génétique? Méthodes, théorie, Paris, CNRS Éditions, 1998, p. 31-60.

11. Elle n'apparaît pas dans le premier manuscrit où manque une feuille.

12. IMEC, fonds Marie Darrieussecq, $849 \mathrm{DRQ} / 32$.

13. IMEC, fonds Marie Darrieussecq, 849DRQ/32.

14. Expression de Marie Darrieussecq sur une feuille volante - une feuille manuscrite recto/ verso, écrite au bic rouge -, IMEC, fonds Marie Darrieussecq, 849DRQ/32.

15. Entretien avec Marie Darrieussecq déjà cité.

16. IMEC, fonds Marie Darrieussecq, 849DRQ/10/7.

17. Entretien avec Marie Darrieussecq déjà cité.

18. Jean-Marie Constans, «Les falaises du mystère », Pyrénées Magazine, $n^{\circ} 45$, mai-juin 1996, p. 74.

19. Marie Darrieussecq, Le Mal de mer (1999), Paris, Gallimard, coll. « Folio », 2001, p. 103.

20. Marie Darrieussecq, White (2003), Paris, Gallimard, coll. « Folio », 2005, p. 130.

21. Marie Darrieussecq, Le Pays (2005), Paris, Gallimard, coll. « Folio », 2007, p. 73, 147 et 219.

22. Campagne Hermès (IMEC, fonds Marie Darrieussecq, 849DRQ/12/13).

23. Voir Le Pays, op. cit., p. 147.

24. Voir Le Pays, op. cit., p. 73.

25. Respectivement pages 38-39, 44 et $5-5 \mathrm{II}^{\mathrm{e}}$ partie des trois tapuscrits (IMEC, fonds Marie Darrieussecq, 849DRQ/6).

26. Entretien avec Marie Darrieussecq déjà cité. 


\section{RÉSUMÉS}

Les documents d'archives de Marie Darrieussecq révèlent que la science et la science-fiction jouent un rôle matriciel dans la genèse de son œuvre. À travers l'étude de sources sciencefictionnelles présentes dans les carnets de lecture de jeunesse, puis du traitement réservé à l'iridium, une donnée scientifique récurrente dans la création de l'autrice, nous analysons comment Marie Darrieussecq se livre à une poétique de l'escamotage selon un continuum qui s'échelonne de la suppression totale du matériau extérieur jusqu'à sa présence diffuse masquée par un travail de récriture. Cette dynamique exogénétique, qui réinterroge la notion de propriété littéraire ou scientifique, invite à reconsidérer le sens de certaines des œuvres publiées. Elle atteste en outre que si l'imaginaire de l'écrivaine est bien hanté par des données scientifiques et science-fictionnelles, ces dernières sont toujours travaillées sur le mode de la minoration, conformément à une poétique qui se veut proche du réel.

Marie Darrieussecq's archival material reveals that science and science fiction play a seminal role in the genesis of her work. By studying the science-fictional sources present in the notebooks of her youth, and also her treatment of iridium, a recurrent chemical element in the author's creation, we analyse how Marie Darrieussecq's writing reflects a poetics of withdrawal, which follows a continuum that ranges from total suppression of outer material to its diffuse presence masked by rewriting. This exogenetic dynamic, which reinterprets the notion of literary or scientific property, leads us to reconsider the meaning of some of her published works. It further testifies to the fact that if her imagination is haunted by scientific and science-fictional data, the latter are always dealt with in a minor mode, in keeping with a poetics that keeps close to reality.

Aus den Archivdokumenten von Marie Darrieussecq geht hervor, dass Wissenschaft und ScienceFiction bei der Entstehung ihres Werks eine Matrixrolle spielen. Durch das Studium von ScienceFiction-Quellen, die in Kindernotizbüchern zu finden sind, und die Behandlung vom chemischen Element Iridium, einer wiederkehrenden wissenschaftlichen Tatsache im Schaffen der Autorin, analysieren wir, wie Marie Darrieussecq sich einer Poetik des Eskapismus entlang eines Kontinuums hingibt, das von der totalen Unterdrückung des äußeren Materials bis zu seiner diffusen, durch Umschreiben maskierten Präsenz reicht. Diese exogenetische Dynamik, die den Begriff des literarischen oder wissenschaftlichen Eigentums neu untersucht, lädt uns ein, die Bedeutung einiger der veröffentlichten Werke neu zu überdenken. Es zeigt auch, dass, wenn die Phantasie des Schriftstellers tatsächlich von wissenschaftlichen und Science-Fiction-Daten verfolgt wird, letztere immer im Modus der Untertreibung bearbeitet werden, gemäß einer Poetik, die darauf abzielt, der Realität nahe zu sein.

Los documentos de archivo de Marie Darrieussecq ponen de manifiesto que la ciencia y la cienciaficción juegan un papel matriz en la génesis de su obra. A través del estudio de las fuentes ciencia-ficcionales presentes en sus carnets de lectura juveniles y del tratamiento reservado al iridio, un dato científico recurrente en la creación de la autora, analizamos como Marie Darrieussecq se entrega a una poética del escamoteo según un continuum que se escalona de la supresión total del material exterior hasta su presencia difusa ocultada por un trabajo de reescritura. Esta dinámica exogenética, que cuestiona una vez más la noción de propiedad 
literaria o científica, promueve una reconsideración del sentido de algunas obras publicadas, demostrando, por otra parte, que si el imaginario de la escritora esta poblado de datos científicos y ciencia-ficcionales, éstos aparecen siempre reelaborados con una perspectiva reductiva, conforme a una poética que pretende estar en las inmediaciones de lo real.

Os documentos de arquivo de Marie Darrieussecq revelam que a ciência e a ficção científica desempenham um papel matricial na génese da sua obra. Através do estudo de fontes de ciênciaficcional presentes nos seus cadernos de leitura de juventude e do tratamento reservado ao irídio, um dado científico recorrente na criação da autora, analisamos como Marie Darrieussecq se entrega a uma poética de camuflagem segundo um continuum que vai desde a supressão total do material exterior até à sua presença difusa camuflada por um trabalho de reescrita. Esta dinâmica exogenética, que volta a questionar a noção de propriedade literária ou científica, convida a reconsiderar o sentido de algumas das obras publicadas. Atesta ainda que, se o imaginário da escritora é assombrado por dados científicos e de ciência-ficcional, estes últimos são sempre trabalhados em menor escala, em conformidade com uma poética que se quer próxima do real.

I documenti d'archivio di Marie Darrieussecq rivelano il ruolo fondamentale della scienza e della fantascienza nella genesi della sua opera. Attraverso lo studio delle fonti fantascientifiche presenti nei carnet di lettura giovanili e del trattamento riservato all'iridio (un dato scientifico ricorrente nella creazione dell'autrice), l'articolo analizza il modo in cui Marie Darrieussecq si dedica a una poetica dell'escamotage, secondo un continuum che va dalla cancellazione totale del materiale esterno fino alla sua presenza diffusa, mascherata da un lavoro di riscrittura. Questa dinamica esogenetica, che interroga la nozione di proprietà letteraria o scientifica, invita a riconsiderare il senso di alcune delle sue opere edite, dimostrando inoltre che se l'immaginario della scrittrice è ossessionato dai dati scientifici e fantascientifici, questi ultimi sono sempre trattati in modo minore, conformemente a una poetica che si vuole vicina alla realtà.

\section{AUTEURS}

\section{KARINE GERMON}

KARINE GERMONI est maîtresse de conférences en Langue française à la faculté des Lettres de Sorbonne Université. Stylisticienne, elle est spécialiste de l'œuvre de Beckett et ses domaines de recherche portent sur : la langue (orthographe et ponctuation) ; la stylistique auteuriste dans la prose narrative des $\mathrm{XX}-\mathrm{XXI}^{\mathrm{e}}$ siècles ; les discours rapportés ; les configurations formelles du repentir ; la poétique des genres et l'écriture au second degré dans le roman et le théâtre contemporains ; l'editing, la génétique et l'énonciation de la fonction éditoriale.

\section{ÉLISE NOTTET-CHEDEVILLE}

Élise Nottet-Chedeville est agrégée de lettres modernes et ATER à la faculté des Lettres de Sorbonne Université. Doctorante au sein de l'équipe d'accueil STIH, elle achève une thèse sur les poèmes de Jean Genet sous la direction de Jacques Dürrenmatt et s'intéresse plus largement à la génétique textuelle, à la stylistique et à la littérature des $\mathrm{xx}^{\mathrm{e}}$ et $\mathrm{xxi}^{\mathrm{e}}$ siècles. Elle a travaillé dernièrement sur Marie Darrieussecq, sur les écritures évolutives et sur les discours rapportés louches et atypiques. 